\title{
EL TRABAJO COOPERATIVO COMO INSTRUMENTO PARA CONSTRUIR LA EDUCACIÓN PATRIMONIAL ${ }^{1}$ \\ COOPERATIVE WORK AS AN INSTRUMENT TO BUILD HERITAGE EDUCATION
}

\author{
María Pilar Molina Torres \\ pilar.molina@uco.es
}

Facultad de Ciencias de la Educación. Universidad de Córdoba (España)

Recibido: $19 / 04 / 2019$

Aceptado: $23 / 05 / 2020$

\begin{abstract}
Resumen:
El patrimonio cultural y natural ofrece multitud de oportunidades para ser utilizado como recurso didáctico en los diferentes niveles de enseñanza. Por este motivo, el objetivo de este trabajo se ciñe a una línea de investigación centrada en la adquisición de las competencias educativas en el Grado de Educación Primaria. El proyecto curricular estuvo relacionado con la Didáctica de las Ciencias Sociales y la Educación Patrimonial. Siguiendo una metodología investigativa, los participantes, dos grupos de alumnos del citado grado $(n=98)$, realizaron diferentes actividades prácticas que volcaron en un blog de aula con la finalidad de recrear una herramienta educativa adaptada a su futura carrera profesional como docentes. Por último, los resultados de la experiencia han sido productivos y enriquecedores para un alumnado que ha puesto en valor los recursos didácticos que pueden implementar en su práctica real.
\end{abstract}

Palabras clave: aprendizaje en grupo, metodología investigativa, patrimonio cultural, proyecto educativo, tiempo histórico.

\begin{abstract}
:
The cultural and natural heritage offers many opportunities to be used as a teaching resource in the different levels of education. For this reason, the aim of this paper is to follow a line of research focused on the acquisition of educational competences in the Primary Education Degree. The curricular project was related to the Didactics of Social Sciences and Heritage Education. Following a research methodology, the participants, two groups of students of the said grade $(n=98)$, carried out different practical activities that turned into a classroom blog in order to recreate an educational tool adapted to their future professional career as teachers. Finally, the results of the experience have been productive and enriching for a student who has valued the teaching resources that can be implemented in their real practice.
\end{abstract}

Keywords: group learning, investigative methodology, cultural heritage, educational project, historical time.

\footnotetext{
${ }^{1}$ Este trabajo se incluye en el Proyecto de Investigación PGC2018-097481-B-I00, financiado por el Ministerio de Ciencia, Innovación y Universidades de España.
} 


\section{Introducción}

El área de Ciencias Sociales está vinculada con el aprendizaje del contexto cultural, social y cívico en el que el alumnado se desenvuelve. A través de la Didáctica de las Ciencias Sociales se trabajan diversos contenidos que ayudan al alumnado del Grado de Educación Primaria a adquirir conocimientos sobre el mundo que les rodea, incluyendo el estudio del patrimonio cultural de la ciudad donde residen. Es más, en la enseñanza del patrimonio histórico es necesario que la evaluación de las estrategias implementadas permita detectar tanto las fortalezas como las debilidades para mejorar la calidad del aprendizaje (Asensio, 2015). De este modo, se hace necesario que dicha asignatura se trabaje y se enseñe desde la motivación, a través de metodologías y recursos didácticos con los que el alumnado aprenda de manera más lúdica.

En las dos últimas décadas la revisión de la literatura científica sobre el trabajo cooperativo como estrategia didáctica y la educación patrimonial ha aportado cuantiosos estudios (Johnson, Johnson y Holubec, 1999; Pujolàs, 2008; Riera, 2011; García-Cabrera, González y Mérida, 2012; Laal y Laal, 2012; Slavin, 2014; Avello-Martínez y Marín, 2015; Cambil, 2015; Fontal e Ibáñez, 2015; Fontal, 2016; Gillies, 2016; Lata y Castro, 2016; Palomares y Chisvert, 2016; Zariquiey, 2016; Castro y López, 2017; Cuenca, Estepa y Martín, 2017; López, Miralles, Prats y Gómez, 2017; Izquierdo, Asensio, Escarbajal y Rodríguez, 2019).

En este sentido, es una realidad que el alumnado de tercer curso del Grado de Educación Primaria ha adquirido a lo largo de su formación destrezas suficientes para trabajar en equipo y de manera cooperativa para propiciar que todos los compañeros tengan acceso a distintos recursos, lo que favorece la interacción educativa en el aula. De hecho, el trabajo en equipo forma parte del proceso de aprendizaje desarrollando competencias, habilidades y destrezas que refuerzan la afectividad desde el trabajo cooperativo y vivencial (Arias, 2017). Es por ello por lo que la flexibilidad en un cambio metodológico real es lo que favorece la capacidad formativa de los futuros docentes de Educación Primaria (López de Sosoaga, Ugalde, Rodríguez y Rico, 2015) y consolida la implementación de las competencias educativas fomentando su iniciativa personal (Zabala y Arnau, 2014).

Esta formación en competencias mejora y valora la educación patrimonial y el conocimiento social (Domínguez y López, 2017), propiciando, a su vez, la construcción de una ciudadanía comprometida (Cuenca, 2014). También, y desde este enfoque patrimonial, la formación del profesorado de Educación Primaria se ve enriquecida por la enseñanza de metodologías activas como el aprendizaje basado en proyectos y el aprendizaje cooperativo (Vicent y Luna, 2019). Estos conocimientos metodológicos mejoran la formación inicial y continua del alumnado, además de las competencias profesionales y académicas (Payà, Gros, Piqué y Rubio, 2018). Por tanto, para el desarrollo de estas competencias y destrezas se debe innovar y mejorar los procesos de aprendizaje (Navarro, González, López y Contreras, 2019). Es aquí donde el trabajo cooperativo implica que los grupos de trabajo se ayuden, compartan ideas y desarrollen habilidades sociales (Iglesias, González y Fernández-Río, 2017).

Así al diseñar un espacio formativo en la universidad se constituye un factor de referencia en las necesidades profesionales de los alumnos del grado (Cano, 2005). Es conveniente señalar que en el aula los diferentes contextos de aprendizaje enriquecen las decisiones grupales, la adaptación de intereses diversos y la producción de los medios para alcanzar estrategias didácticas. Asimismo, se añade que el desarrollo de tareas y los grupos de colaboración contribuyen para establecer estrategias útiles y con ello reforzar los contenidos curriculares. De hecho, el aprendizaje colaborativo es una herramienta que, a menudo, crea sinergias didácticas y situaciones de interdependencia que promoverán un proceso de enseñanza. Esta idea coincide 
con la afirmación de Avello-Martínez y Marín (2015) cuando destacan que el aprendizaje colaborativo sólo se refiere a una situación en la que se espera (y no se asegura) que se produzcan determinadas formas de interacción que implicarán un aprendizaje.

Actualmente, la implementación de aprendizajes interactivos ha relegado gradualmente la práctica de la metodología tradicional (Cebrián, 2003; Cózar y Sáez, 2017), además de adquirir no solo las competencias clave del currículum de Educación Primaria, sino además las competencias socioemocionales que favorezcan la vida en sociedad de nuestro alumnado y las capacidades de trabajar en equipo (Perrenoud, 2004; Cano, 2005; Escamilla, 2009). Es así como pudimos potenciar las ganas de experimentar y de utilizar otros métodos educativos más innovadores que les permitan incrementar sus habilidades sociales y fortalecer el conocimiento conceptual, la reflexión propia y el análisis de situaciones cercanas a la realidad (Solaz, Sanjosé y Gómez, 2011; Saiz y Rivas, 2012).

Frente a un enfoque de metodología tradicional, una ventaja significativa es el tipo de actividades que se trabajan entablando un entorno de discusión y, sobre todo, de curiosidad e interés por descubrir y experimentar. El conocimiento del territorio y los elementos culturales que lo componen debe tener lugar tanto en un contexto educativo como social y formativo (Sanchidrián, 2011). La educación patrimonial es una prioridad indispensable en un escenario esencialmente globalizado y sin un interés significativo por las culturas que nos precedieron. Es necesario para comprender la gestión del patrimonio que el proceso histórico de una comunidad quede definido en un espacio holístico que conforme una identidad colectiva e individual. De esta manera, se concibe que la enseñanza de la historia y su didáctica sea imprescindible para dar respuesta a las dificultades de aprendizaje de los estudiantes del Grado de Educación Primaria (Prats, 2008; Fontal, Ibáñez, Martínez y Rivero, 2017; Molina, Miralles, Deusdad y Alfageme, 2017). Así pues, es evidente que los docentes se encuentran preparados profesionalmente para abordar en la práctica educativa los conocimientos y actitudes patrimoniales que están implícitas en el desarrollo de las competencias (Salinas, De Benito y Lizana, 2014; Alonso, 2018).

Ahora bien, las estrategias de enseñanza del patrimonio histórico-artístico en Educación Primaria no crean un contacto adecuado para que la combinación patrimonio vs identidad sea un elemento transmitido en la escuela (Cuenca, Estepa y Martín, 2011). Asimismo, el carácter localista de muchos de los recursos didácticos utilizados no es relevante para una interpretación del patrimonio a nivel general. Por tanto, es conveniente planificar un enfoque significativo que abarque las diferentes etapas educativas desde una perspectiva curricular. De hecho, la educación patrimonial se presenta como un elemento educativo que debe ser adquirido por los docentes, y en nuestro caso concreto por los estudiantes del Grado de Educación Primaria (Molina, 2017).

De este modo es beneficiosa para los gestores del patrimonio y los docentes la consolidación de una relación estrecha entre el entorno y la escuela. Al respecto, Martín y Cuenca (2011) apuntan que los gestores tienen un elevado nivel de desarrollo conceptual acerca de la comprensión del patrimonio y la tipología patrimonial, pero sin embargo no son capaces de traducir este conocimiento en propuestas educativas efectivas para la sociedad. Por este motivo, y como se recoge en las siguientes páginas, este acercamiento es posible siempre y cuando la didáctica del patrimonio se fomente tanto en un contexto educativo formal y también en un espacio no formal.

\section{Objetivos del estudio}

La problemática de esta investigación se ajusta a la necesidad de realizar propuestas educativas que integren la visión patrimonial de los estudiantes del Grado de Educación Primaria. De esta 
manera, el objetivo que se plantea es averiguar cuáles son las percepciones de los alumnos en relación con el patrimonio histórico-artístico de su ciudad, Córdoba, en época romana y las repercusiones que la falta de conocimientos curriculares tiene en la implementación universitaria de la materia de Didáctica de las Ciencias Sociales. Para complementar este objetivo general se plantearon una serie de objetivos específicos: (1) fomentar el trabajo cooperativo; (2) estimular el interés por el patrimonio histórico; (3) identificar las actitudes positivas y/o negativas hacia una metodología investigativa y (4) conocer el nivel de implicación en torno a la identidad patrimonial.

En este sentido, y para ejemplificar el desarrollo del proyecto, agrupamos en la siguiente tabla los objetivos además de las competencias educativas y los contenidos que se programaron en la guía docente de la citada asignatura para, a su vez, transponer la afinidad de todos estos elementos con los recogidos en el Real Decreto 126/2014, de 28 de febrero por el que se establece el currículo básico de la etapa educativa y la Orden de 17 de marzo de 2015, por la que se desarrolla el currículo correspondiente a la Educación Primaria en Andalucía.

\begin{tabular}{|c|c|c|}
\hline & $\begin{array}{c}\text { Guía docente (Didáctica de las } \\
\text { Ciencias Sociales en Educación } \\
\text { Primaria) }\end{array}$ & $\begin{array}{c}\text { Real Decreto } 126 / 2014 \text {, de } 28 \text { de } \\
\text { febrero y Orden de } 17 \text { de marzo de } \\
2015\end{array}$ \\
\hline Objetivos generales & $\begin{array}{l}\text {-Determinar la finalidad de la } \\
\text { enseñanza de las Ciencias Sociales } \\
\text { en la Enseñanza Primaria. } \\
\text {-Poner en contacto a los alumnos } \\
\text { con los materiales y recursos } \\
\text { didácticos más adecuados para la } \\
\text { enseñanza de las Ciencias Sociales, } \\
\text { de tal forma que no sólo conozca su } \\
\text { utilización, sino que sean capaces } \\
\text { de elaborar material propio. } \\
\text {-Potenciar la actitud investigadora } \\
\text { de los futuros maestros respecto a } \\
\text { todos los elementos que } \\
\text { intervienen en el proceso de } \\
\text { enseñanza-aprendizaje de las } \\
\text { Ciencias Sociales en la Enseñanza } \\
\text { Primaria. }\end{array}$ & $\begin{array}{l}\text {-Conocer los aspectos fundamentales } \\
\text { de las Ciencias de la Naturaleza, las } \\
\text { Ciencias Sociales, la Geografía, la } \\
\text { Historia y la Cultura. } \\
\text { - Desarrollar hábitos de trabajo } \\
\text { individual y de equipo, de esfuerzo y } \\
\text { de responsabilidad en el estudio, así } \\
\text { como actitudes de confianza en sí } \\
\text { mismo, sentido crítico, iniciativa } \\
\text { personal, curiosidad, interés y } \\
\text { creatividad en el aprendizaje, y } \\
\text { espíritu emprendedor } \\
\text { - Iniciarse en la utilización, para el } \\
\text { aprendizaje, de las Tecnologías de la } \\
\text { Información y la Comunicación } \\
\text { desarrollando un espíritu crítico ante } \\
\text { los mensajes que reciben y elaboran. }\end{array}$ \\
\hline $\begin{array}{l}\text { Competencias } \\
\text { trabajadas }\end{array}$ & $\begin{array}{l}\text { CE10. Reflexionar sobre las } \\
\text { prácticas de aula para innovar y } \\
\text { mejorar la labor docente. Adquirir } \\
\text { hábitos y destrezas para el } \\
\text { aprendizaje autónomo y } \\
\text { cooperativo y promoverlo entre los } \\
\text { estudiantes. } \\
\text { CE11. Conocer y aplicar en las aulas } \\
\text { las tecnologías de la información y } \\
\text { de la comunicación. Discernir } \\
\text { selectivamente la información } \\
\text { audiovisual que contribuya a los } \\
\text { aprendizajes, a la formación cívica } \\
\text { ya la riqueza cultural. } \\
\text { CM5.7. Desarrollar y evaluar } \\
\text { contenidos del currículum } \\
\text { mediante recursos didácticos }\end{array}$ & $\begin{array}{l}\text {-El carácter global del área de Ciencias } \\
\text { sociales hace que contribuya en } \\
\text { mayor o menor medida, al desarrollo } \\
\text { de la mayoría de las competencias } \\
\text { claves. } \\
\text { - Contribuye también de forma } \\
\text { relevante, a la competencia digital. } \\
\text { Leer un mapa, interpretar un gráfico, } \\
\text { observar un fenómeno o utilizar una } \\
\text { fuente histórica, exige el uso de } \\
\text { procedimientos diferenciados de } \\
\text { búsqueda, selección, organización e } \\
\text { interpretación, que son objeto de } \\
\text { aprendizaje en esta área. } \\
\text { - El aprendizaje de las Ciencias sociales } \\
\text { contribuirá al desarrollo de la } \\
\text { competencia aprender a aprender, }\end{array}$ \\
\hline
\end{tabular}




\begin{tabular}{|l|l|l|}
\hline & $\begin{array}{l}\text { apropiados y promover las } \\
\text { competencias correspondientes en } \\
\text { los estudiantes. }\end{array}$ & $\begin{array}{l}\text { mediante el desarrollo de técnicas } \\
\text { para tratar la información: la } \\
\text { realización de resúmenes, esquemas } \\
\text { o mapas conceptuales que resultan } \\
\text { especialmente útiles en los procesos } \\
\text { de aprendizaje. }\end{array}$ \\
\hline $\begin{array}{l}\text { Contenidos } \\
\text { seleccionados }\end{array}$ & $\begin{array}{l}\text { Bloque 2: El aprendizaje de las } \\
\text { Ciencias Sociales. } \\
\text { Bloque 3: Estrategias y desarrollo } \\
\text { de habilidades, técnicas y recursos } \\
\text { para la enseñanza de las Ciencias } \\
\text { Sociales. }\end{array}$ & $\begin{array}{l}\text {-Iniciación al conocimiento científico y } \\
\text { su aplicación en las Ciencias Sociales } \\
\text {-Utilización de las tecnologías de la } \\
\text { información y la comunicación para } \\
\text { buscar y seleccionar información y } \\
\text { presentar conclusiones. } \\
\text {-Uso y utilización correcta de diversos } \\
\text { materiales con los que se trabaja. } \\
\text {-Utilización de estrategias para } \\
\text { potenciar la cohesión del grupo y el } \\
\text { trabajo cooperativo. }\end{array}$ \\
\hline
\end{tabular}

Tabla 1. Diferentes apartados del proyecto que se relacionan con la guía docente de Didáctica de las Ciencias Sociales en el curso 2017/2018. Fuente: Elaboración propia.

\section{Metodología}

\subsection{Participantes}

La muestra de estudio contó con 98 estudiantes del Grado de Educación Primaria de la Universidad de Córdoba de los cuales 46 fueron hombres (46.9\%) y 52 mujeres (53.1\%), que cursaron la asignatura de Didáctica de las Ciencias Sociales en el tercer curso de dicha titulación. Los participantes tenían edades comprendidas entre los 21 y los 23 años. La selección de la población que no tuvo un carácter probabilístico optó por aquellos grupos de estudiantes que estuvieron dispuestos a implicarse en el desarrollo del proyecto y en la investigación educativa.

\subsection{Procedimiento}

La implementación de la propuesta se fundamentó con un enfoque investigador, basado en una metodología de indagación (García-González y Gabriela, 2014; Gómez, Ortuño y Miralles, 2018) y el desarrollo de las competencias docentes y profesionales que definen la estrategia metodológica a través de un itinerario didáctico por la ciudad y sus vestigios romanos que aún se hallan in situ. Como recurso educativo estos itinerarios se convierten en un elemento multidisciplinar que permite al alumnado y al docente mantener una interacción de forma directa con el medio (Ávila, 2003; Vilarrasa, 2003). Según afirma Cambil (2015), los itinerarios didácticos por sus grandes ventajas educativas son una de las estrategias más utilizadas en la enseñanza y aprendizaje del patrimonio cultural en la que se utiliza la ciudad como recurso. Al respecto García de la Vega (2004) señala que el itinerario es un recurso motivador, útil y valioso para el alumno, ya que permite desarrollar el conocimiento (crea y consolida conceptos e ideas) y conduce a valorar el significado del paisaje. Para Liceras (2018), los itinerarios y las prácticas de campo son aquellas actividades que deben desarrollarse preferentemente en la programación docente con el objetivo de crear un aprendizaje significativo en el alumnado.

Por otra parte, la investigación y la experimentación también formaron parte de la activa construcción de conocimientos históricos y patrimoniales. En la enseñanza del patrimonio la aplicación de una metodología investigativa es una alternativa metodológica que, en la práctica, facilita la búsqueda de una o varias respuestas a un interrogante que provoca una implicación directa en la propuesta didáctica que se plantea. Es así como a través de las diferentes fases del proceso se adquieren de manera espontánea las competencias educativas (Hernández, 
Fernández y Baptista, 2010). De este modo, el aprendizaje investigativo ofrece a los estudiantes una aproximación a las características edilicias y administrativas de una colonia romana (Molina, 2018).

En este proceso investigativo se utilizaron diferentes técnicas que les permitió recoger al alumnado la información que luego se ha de analizar a través de las narrativas personales, el portafolio, los cuestionarios, la observación, los dosieres de prácticas. Asimismo, a través de la investigación-acción conseguimos:

- Fomentar el trabajo colaborativo y grupal para dar respuesta a sus inconvenientes de una manera crítica y reflexiva.

- Facilitar al alumnado un rol investigador que les capacite para su desarrollo profesional y les proporcione autonomía personal.

- Promover una mayor conciencia formativa entre los estudiantes que buscan nuevas formas de rediseñar y concebir sus experiencias educativas.

El método de enseñanza utilizado en el desarrollo y diseño de la experiencia estuvo adaptado a las características de los alumnos que componen el grupo-clase. Para evitar las clases magistrales, se proponen una serie de talleres didácticos centrados en el aprendizaje por descubrimiento para que el alumnado investigue sobre la civilización romana. Los agrupamientos fueron flexibles de manera que pudieran trabajar en gran grupo, pequeños grupos y por parejas, fomentando con ello el trabajo cooperativo, el ajuste de los ritmos y espacios y la variedad de recursos didácticos (Pujolàs, 2008).

Los participantes del proyecto siguieron una secuencia de una sesión semanal práctica de cuatro horas y media durante tres semanas, con un desdoble de tres pequeños grupos de aproximadamente veinte alumnos a los que se dedicó una hora y media. Para las diferentes etapas los estudiantes decidieron la manera más adecuada de temporalizar la propuesta:

Fase 1. Planificación del trabajo (una sesión): el reparto y planificación de las tareas en pequeños grupos de cinco alumnos. La división de funciones se organiza de manera que todos los participantes tengan unos roles que pueden ir rotando durante las sesiones de trabajo. En una primera sesión inicial se plantearon varias preguntas de introducción al tema: ¿Qué sabemos? ¿Qué queremos investigar? ¿Cómo lo vamos a lograr?

Fase 2. Implementación de la propuesta (tres sesiones): establecidos los roles, pasamos a la ejecución del blog de aula. En pequeños grupos investigaron en la web diversos formatos de blog de aula a través de tutoriales formativos que se colgaron en Moodle y que dieron lugar a la resolución de dudas y la confección de esta herramienta digital.

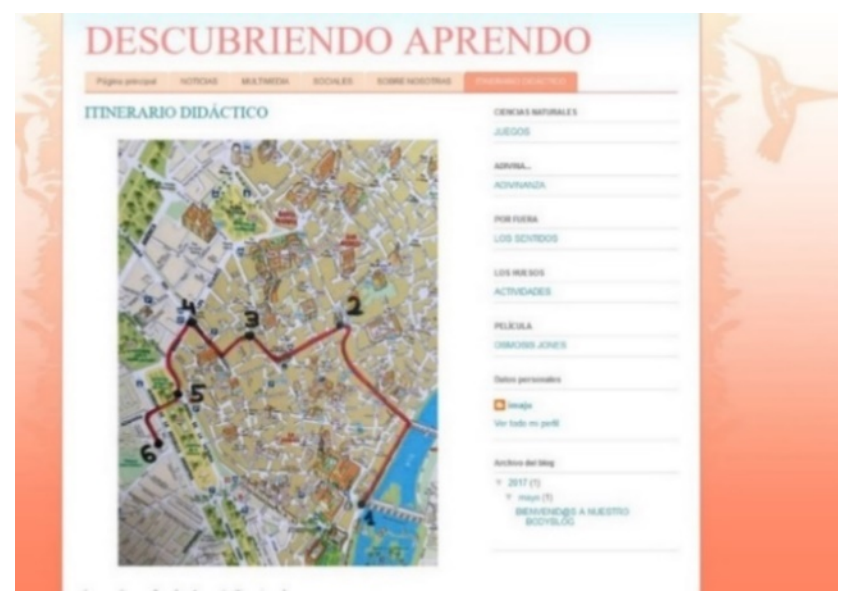

Figura 1. Blog de aula con el itinerario didáctico diseñado por el alumnado. Fuente: http://descubriendo3.blogspot.com/p/blog-page 16.html 
La planificación se configuró en torno a los conocimientos previos y a la contextualización histórica del alumnado en la Corduba romana. De este modo, programamos un recorrido por seis temáticas que se trabajaron previamente dentro del aula en tres sesiones, y tras las tareas iniciales de investigación mediante un itinerario que se planificó en una sesión práctica (fase 4): (1) las actividades económicas, (2) las creencias religiosas, (3) el espacio urbano y administrativo de la colonia, (4) la delimitación territorial de la urbe y las murallas, (5) los cultos funerarios y, por último, (6) los edificios de espectáculos. Por otra parte, en lo que respecta a las paradas que defendieron como expertos en la materia, el itinerario siguió el orden que se recoge (figura 1):

1.- Puerta del Puente (Puerto romano, economía y comercio)

2.- Templo de culto imperial en la calle Claudio Marcelo (Religión y devociones)

3.- Plaza de las Tendillas (Foro romano y administración municipal)

4.- Esquina de Cajasur frente al Corte Inglés (Muralla y arquitectura doméstica)

5.- Mausoleos funerarios de Puerta Gallegos (Mundo funerario)

6.- Edificio rectoral de la UCO (Anfiteatro, ludi romanos)

En este sentido, las investigaciones previas que organizamos antes de la salida y que dieron sentido a las explicaciones en dichas paradas, se volcaron en un blog de aula que utilizaron para compartir y actualizar las actividades que pusieron en práctica en las exposiciones. Con el fin de que dieran forma a lo aprendido y explicado en clase, se pidió a los alumnos que reflexionaran sobre el enfoque que darían a la experiencia didáctica y elaboraran su propia propuesta comparando los monumentos actuales integrados patrimonialmente en la ciudad con los documentados en época romana.

Fase 3. Investigación y experimentación didáctica (tres sesiones): la investigación sobre la temática a trabajar siguió un procedimiento enriquecedor mediante el intercambio de ideas y la colaboración grupal para recopilar diversos recursos virtuales como mapas interactivos, vídeos didácticos, webquest. La selección de estos materiales supuso un acercamiento entre los pequeños grupos de trabajo que fomentó la acción colaborativa para el volcado de contenidos y el diseño de los talleres experimentales que versarán sobre la familia romana, el urbanismo, las creencias y la economía de la colonia.

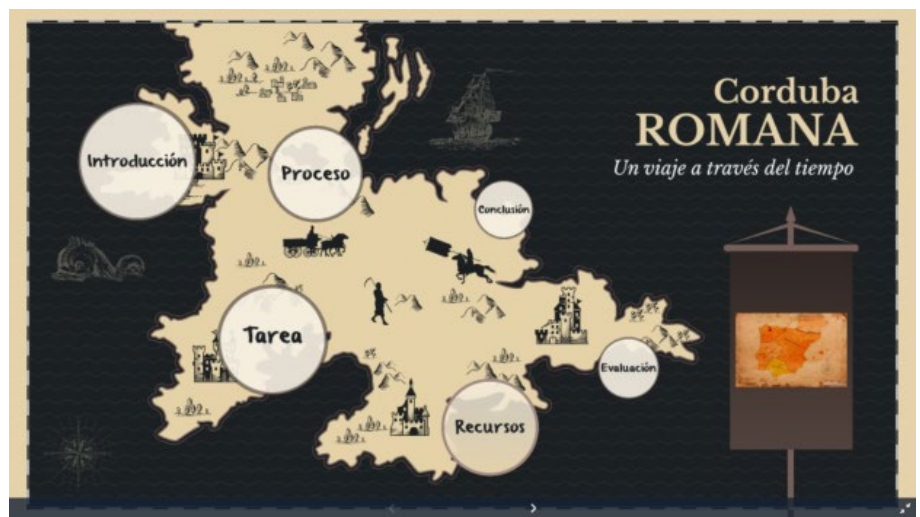

Figura 2. Prezi sobre Corduba romana.

Fuente: https://prezi.com/view/OJQjdDQuufRGJI10zROy/ 


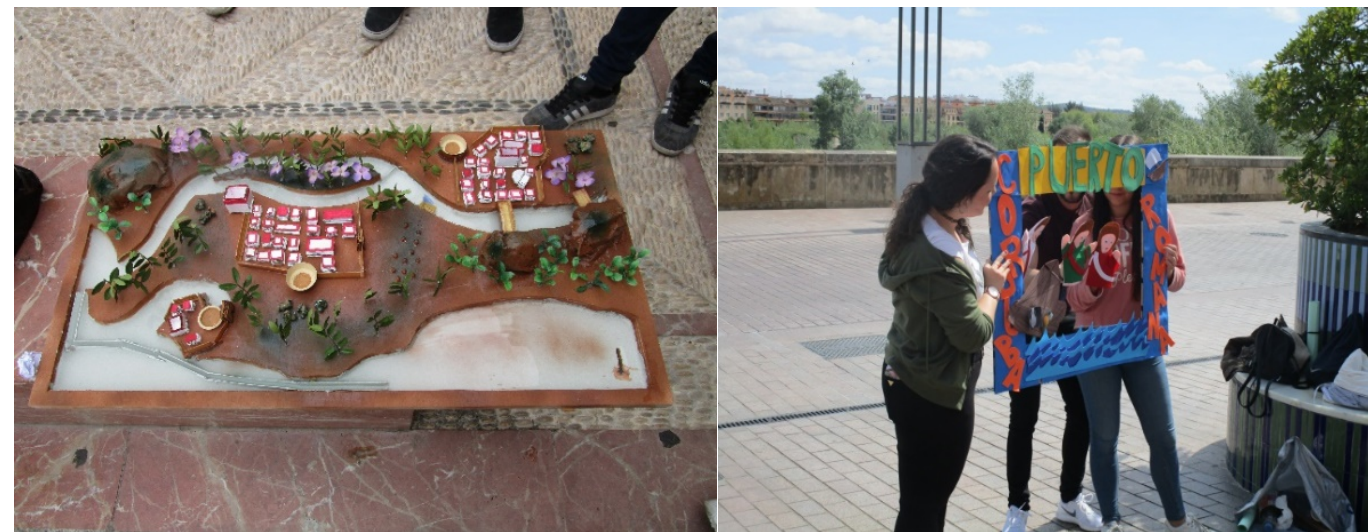

Figuras 3-4. Talleres didácticos con la reconstrucción de una ciudad romana y un guiñol sobre el comercio fluvial en Corduba.

Fase 4. Síntesis y recapitulación de ideas (una sesión): la actividad final se centró en realizar por grupos una presentación en Genially con los resultados de la temática investigada. Para complementar los resultados, pusimos en práctica el itinerario didáctico con una salida por la Córdoba romana que nos permitió cotejar los trabajos realizados y volcados en el blog de aula, y hacer un balance final de la experiencia. Seguidamente, se delimitó el itinerario educativo dentro del entramado urbano que el alumnado diseñó y materializó durante toda la fase final. Los alumnos como expertos en las diferentes materias asignadas expusieron sus investigaciones en las paradas establecidas dentro de la ruta urbana. Por último, valoramos el proyecto a través de rúbricas de evaluación.

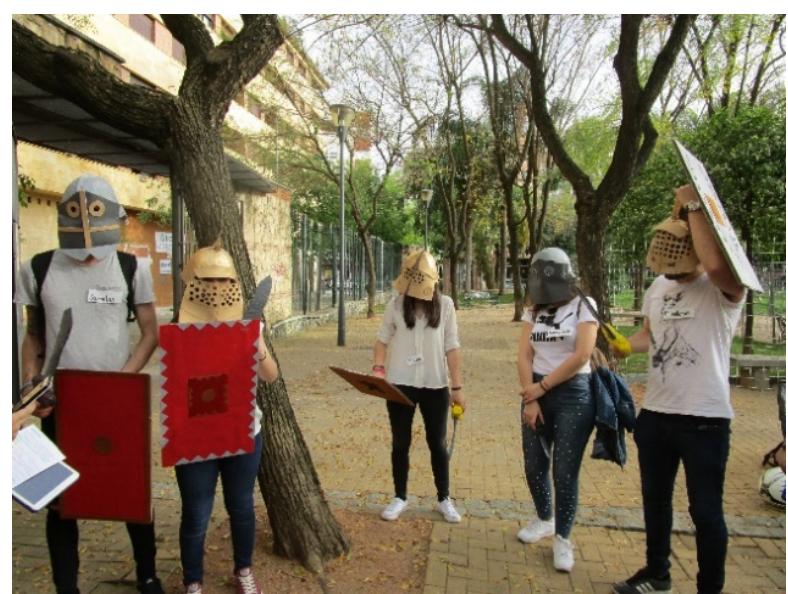

Figura 4. Itinerario por el anfiteatro romano y exposición de la parada correspondiente a los espectáculos. Alumnos caracterizados como gladiadores en las cercanías del monumento in situ.

\subsection{Diseño de investigación e instrumento de recogida de información}

Para el diseño de esta investigación que siguió un modelo cuantitativo tipo cuestionario ha sido necesario tener en cuenta qué método o alternativas son las más adecuadas para el diseño del instrumento de recogida de datos (Cohen y Manion, 2002).

Se optó por una batería de preguntas con un total de dieciocho ítems agrupados en tres categorías: (1) Heteroevaluación de la docente, (2) Autoevaluación del alumnado, y (3) Evaluación de los talleres didácticos. Para la recogida de la información se utilizó un cuestionario diseñado ad hoc para estudiar las categorías 2 y 3, que contempla tres posibles respuestas: Sí, No, A veces, además de un apartado de comentarios individual (tabla 3 ). Junto a este instrumento de recogida de información se configura otra escala de valoración para evaluar la categoría 1 , y que se compone de cuatro valores: $A, B, C \circ D$, que oscilan desde el más positivo 
A hasta el más negativo $D$ (tabla 2). Ambos instrumentos nos han permitido obtener información acerca de los conocimientos patrimoniales del alumnado y su contacto directo con el trabajo cooperativo.

\section{Resultados}

Para los resultados de las tablas que se analizan a continuación se han utilizado dos tipos de evaluación. Por un lado, destaca la heteroevaluación que realiza la profesora al alumnado en los talleres educativos. Los resultados de la tabla 2 muestran un recuento significativo y positivo hacia la puesta en práctica de estos talleres que favorecen el aprendizaje de los conocimientos históricos y el trabajo grupal.

\begin{tabular}{|c|c|c|c|c|}
\hline Alumno/a & & & & \\
\hline Indicadores & A & B & C & $\mathrm{D}$ \\
\hline $\begin{array}{l}\text { Relaciona adecuadamente los contenidos prácticos y teóricos } \\
\text { del proyecto. }\end{array}$ & 91 & 5 & 1 & 1 \\
\hline $\begin{array}{l}\text { Aporta ideas creativas al grupo y participa en las tareas grupales } \\
\text { e individuales. }\end{array}$ & 87 & 8 & 0 & 3 \\
\hline $\begin{array}{l}\text { Identifica los edificios más destacados de la ciudad y su } \\
\text { funcionalidad en época romana. }\end{array}$ & 93 & 2 & 3 & 0 \\
\hline $\begin{array}{l}\text { Va avanzando en el camino hacia un aprendizaje competencial y } \\
\text { autónomo. }\end{array}$ & 95 & 2 & 0 & 1 \\
\hline $\begin{array}{l}\text { Maneja herramientas investigativas para dar respuesta a las } \\
\text { distintas cuestiones sobre el tema que se trabaja. }\end{array}$ & 89 & 3 & 6 & 0 \\
\hline Es capaz de trabajar en grupo. & 86 & 8 & 4 & 0 \\
\hline Muestra interés por aprender durante la propuesta. & 90 & 3 & 5 & 0 \\
\hline
\end{tabular}

Tabla 2. Escala de valoración del profesor hacia los alumnos y recuento de las respuestas. Fuente: elaboración propia. 


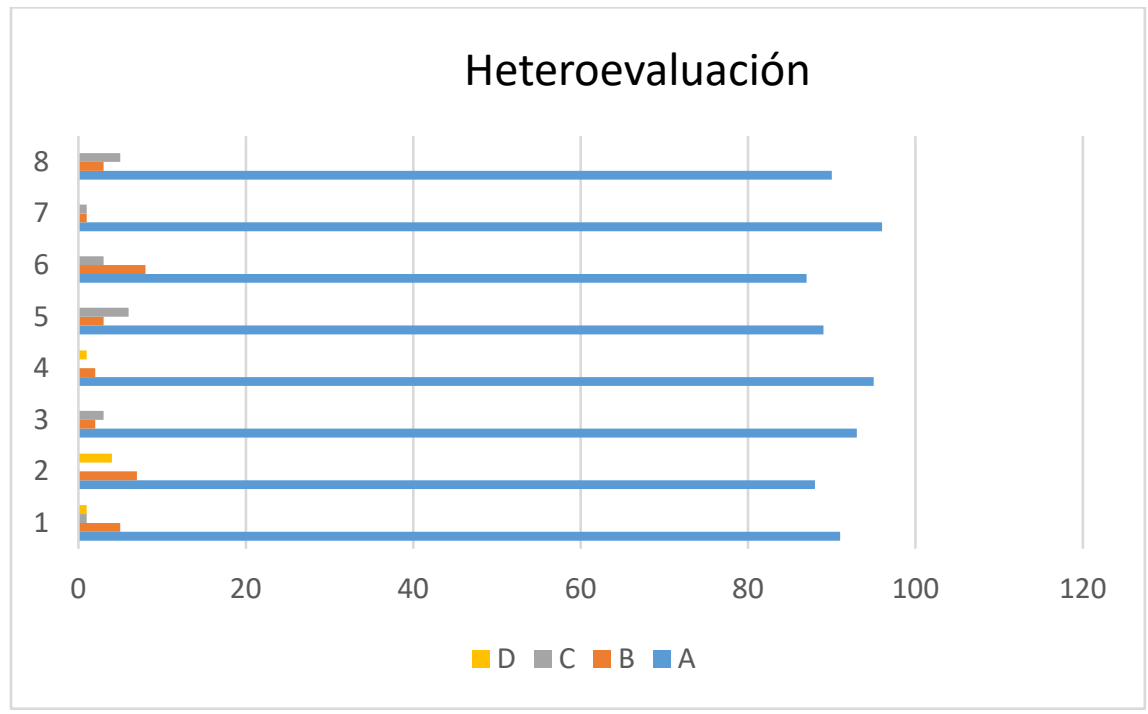

Gráfica 1. Heteroevaluación de la docente. Fuente: elaboración propia.

Por otra parte, acometimos la autoevaluación del alumnado durante los talleres implementados. En la valoración se consideraron las actuaciones personales, las grupales y los recursos que emplearon. Dicha escala de valoración contó con un apartado de comentarios individual (tabla 3). De nuevo, los resultados arrojan una respuesta positiva hacia la ejecución de un proyecto con un método de enseñanza activo y el trabajo por talleres como una alternativa para aprender del patrimonio.

\begin{tabular}{|c|c|c|c|}
\hline Mi desempeño en el proyecto & Sí & A veces & No \\
\hline He propuesto ideas novedosas a mi grupo & 95 & 0 & 3 \\
\hline He colaborado en las tareas grupales & 94 & 4 & 0 \\
\hline He prestado atención a las recomendaciones de la docente & 88 & 9 & 1 \\
\hline He participado en las actividades asignadas & 95 & 1 & 2 \\
\hline Me he implicado en la propuesta didáctica & 92 & 4 & 2 \\
\hline $\begin{array}{l}\text { Me gustaría participar en otro proyecto con la misma } \\
\text { metodología }\end{array}$ & 96 & 0 & 2 \\
\hline \multicolumn{4}{|l|}{ Comentarios } \\
\hline Los talleres educativos & Sí & & No \\
\hline Los talleres didácticos me han interesado & 95 & & 3 \\
\hline He aprendido más sobre mi entorno y mi ciudad & 94 & & 4 \\
\hline Esperaba algo distinto & 53 & & 45 \\
\hline Me gustaría hacer algún cambio & 17 & & 81 \\
\hline Comentarios & & & \\
\hline
\end{tabular}

Tabla 3. Escala de valoración de los alumnos y recuento de las respuestas. Fuente: elaboración propia. 


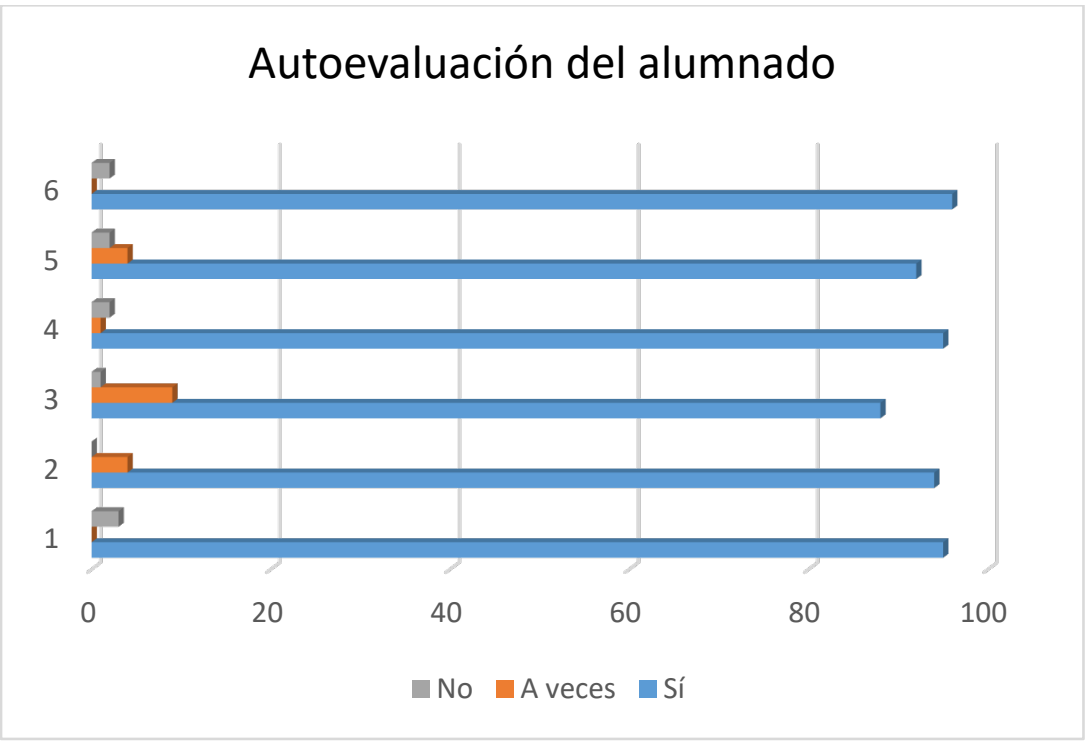

Gráfica 2. Autoevaluación del alumnado. Fuente: elaboración propia.

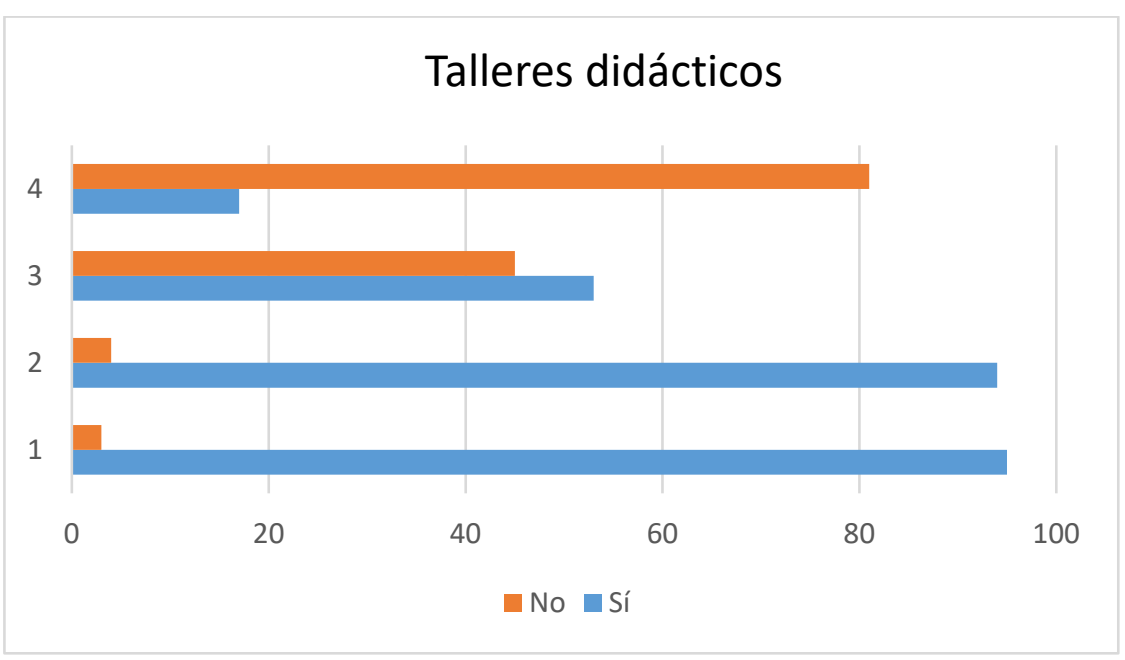

Gráfica 3. Evaluación de los talleres didácticos. Fuente: elaboración propia.

Por último, la evaluación de nuestro proyecto educativo la entendemos como una fórmula de revisión permanente del trabajo desarrollado, una evaluación formativa que además nos permita incorporar sus resultados en el propio proceso o bien nos ayude a plantear más eficazmente nuevas propuestas. Para ello, establecemos medidas como elemento de mejora del proceso de enseñanza aprendizaje, no sólo para comprobar si los alumnos han adquirido las competencias que se querían alcanzar, sino también de la labor docente y la idoneidad de la propia propuesta.

\begin{tabular}{|l|l|}
\hline \multicolumn{1}{|c|}{ Fortalezas } & \multicolumn{1}{c|}{ Debilidades } \\
\hline Experiencia vivencial & Escasa formación del profesorado \\
\hline Aprendizaje significativo & Carencias curriculares acerca del patrimonio local \\
\hline Actividades dinámicas & $\begin{array}{l}\text { Necesidad de mejorar el comportamiento y las } \\
\text { actitudes grupales }\end{array}$ \\
\hline Proximidad al entorno & $\begin{array}{l}\text { Número reducido de docentes que participan en } \\
\text { la experiencia }\end{array}$ \\
\hline Motivación (alumnado/docentes) & $\begin{array}{l}\text { Falta de organización en la planificación del } \\
\text { itinerario }\end{array}$ \\
\hline
\end{tabular}

Tabla 4. Fortalezas y debilidades de la implementación del itinerario didáctico.

Fuente: elaboración propia. 


\section{Discusión y conclusiones}

En líneas generales, varios han sido los beneficios del proyecto que han permitido alcanzar todos los objetivos fijados al comienzo de la propuesta. Entre ellos destacan el diseño de actividades interdisciplinares para alcanzar un aprendizaje significativo, la implementación de una nueva metodología de indagación en el aula y fuera de ésta, la mejora de la coordinación entre los equipos multidisciplinares implicados en la propuesta, la renovación de las prácticas de aula para innovar y mejorar la labor docente, la unificación del trabajo cooperativo en la asignatura de Didáctica de las Ciencias Sociales y, por último, el estímulo hacia la investigación y el trabajo grupal como opción metodológica en la enseñanza universitaria.

En esta línea, Lata y Castro (2016) estudiaron la repercusión que el aprendizaje cooperativo tiene en la escuela y la adquisición de valores que mejoraron la convivencia del alumnado. Entre las ventajas de esta estrategia metodológica de aprendizaje se encuentra la alfabetización mediática (Palomares y Chisvert, 2016). De hecho, la puesta en práctica de una clase cooperativa permite poner a prueba las ideas de los alumnos y obtener retroalimentación del proceso educativo (Johnson, Johnson y Holubec, 1999). Una década después Izquierdo, Asensio, Escarbajal y Rodríguez (2019) subrayan la importancia del trabajo grupal para alcanzar las distintas fases de una tarea y consensuar soluciones a un proceso en común que todos aceptan $y$ del que se hacen responsables.

Como indica Gillies (2016), el papel del docente en el desarrollo de la cooperación grupal incluye estructurar los grupos y las tareas para que los estudiantes entiendan lo que se espera que hagan y cómo deben comportarse. No obstante, con esta experiencia las limitaciones se centraron, especialmente, en la desconfianza del alumnado hacia la metodología implementada, lo que suscita una falta de motivación del grupo al emprender un proyecto del que desconoce la temática y las fases de un proceso esencialmente investigativo al que no está familiarizado en otras asignaturas. En cualquier caso, un proyecto lúdico y productivo no depende solo de una temática apropiada o un enriquecedor producto final, también la coordinación o una significativa gestión de recursos, entre otras cuestiones, mantienen un adecuado proyecto en el tiempo (Riera, 2011).

De esta manera, el trabajo por competencias es una pieza clave dentro del proceso de enseñanza-aprendizaje en la escuela y su implementación aúna el aprendizaje vivencial y significativo en la práctica docente del trabajo cooperativo. Kocabas y Gokce (2017), inciden en la necesidad de conocer los problemas que tienen los docentes con la implementación del aprendizaje cooperativo como método de aprendizaje activo y democrático. Asimismo, los autores confirman que esta falta de preparación la han obtenido de su formación universitaria. De hecho, la situación que muestra el recuento de respuestas de las escalas de valoración nos confirma el interés de los estudiantes en una metodología investigativa con la que no están acostumbrados a evaluar ni a trabajar.

\section{Referencias bibliográficas}

Alonso, A. (2018). Aprendizaje Basado en Proyectos para el desarrollo de la Competencia Digital Docente en la Formación Inicial del Profesorado. Relatec: Revista Latinoamericana de Tecnología Educativa, $17(1), 9-24$.

Arias, L. (2017). El aprendizaje por proyectos: Una experiencia pedagógica para la construcción de espacios de aprendizaje dentro y fuera del aula. Revista Ensayos Pedagógicos, 12(1), 51-68.

Asensio, M. (2015). El aprendizaje natural, la mejor vía de acercarse al patrimonio. Educatio Siglo XXI, 33(1), 55-82.

Avello-Martínez, R. y Marín, V. (2015). La necesaria formación de los docentes en aprendizaje colaborativo. Profesorado. Revista de curriculum y formación del profesorado, 20(3), 687-713. 
Ávila, R. M. (2003). La función del itinerario en la enseñanza y el aprendizaje del Patrimonio HistóricoArtístico. Íber: Didáctica de las ciencias sociales, geografía e historia, 36, 36-47.

Cano, E. (2005). Cómo mejorar las competencias de los docentes. Barcelona: Graó.

Cambil, M. E. (2015). La ciudad como recurso para la enseñanza aprendizaje del patrimonio cultural. Opción. Revista de Ciencias Humanas y Sociales, 31, 295-319.

Castro, B. y López, R. (2017). ¿Para qué sirve el patrimonio cultural? En R. López, P. Miralles, J. Prats (Dirs.) y C. Gómez (Coord.), Enseñanza de la historia y competencias educativas (pp. 67-85). Barcelona: Grao.

Cebrián, M. (coord.) (2003). Enseñanza virtual para la innovación universitaria. Madrid: Narcea.

Cohen, L. y Manion, L. (2002). Método de investigación educativa. Madrid: Editorial la Muralla.

Cózar, R. y Sáez, J. M. (2017). Realidad aumentada, proyectos en el aula de primaria: experiencias y casos en Ciencias Sociales. EDMETIC, 6(1), 165-180.

Cuenca, J. (2014). El papel del patrimonio en los centros educativos: hacia la socialización patrimonial. Tejuelo, 19, 76-96.

Cuenca, J. M., Estepa, J. y Martín, M. J. (2011). El patrimonio cultural en la educación reglada. Revista de Patrimonio cultural de España. Patrimonio y Educación, 5, 45-57.

(2017). Patrimonio, educación, identidad y ciudadanía. Profesorado y libros de texto en la enseñanza obligatoria. Revista de Educación, 375, 136-157.

Domínguez, A. y López, R. (2017). Formación de maestros y educación patrimonial. Estudios pedagógicos, 43(4), 49-68.

Escamilla, A. (2009). Las competencias en la programación del aula. Barcelona: Grao.

Fontal, O. e Ibáñez, A. (2015). Estrategias e instrumentos para la educación patrimonial en España. Educatio siglo XXI: Revista de la Facultad de Educación, 33,15-32 (Ejemplar dedicado a: Actualidad y tendencias en Educación Patrimonial).

Fontal, O. (2016). Educación patrimonial: retrospectiva y prospectivas para la próxima década. Estudios Pedagógicos, 42(2), 415-436.

Fontal, O., Ibáñez, A., Martínez, M. y Rivero, M. P. (2017). El patrimonio como contenido en la etapa de Primaria: del currículum a la formación de maestros. Revista electrónica interuniversitaria de formación del profesorado, 20(2), 79-94.

García-Cabrera, M. M., González, I. y Mérida, R. (2012). Validación del cuestionario de evaluación ACOES. Análisis del trabajo cooperativo en educación superior. Revista de Investigación Educativa, 30(1), 87-109.

García de la Vega, A. (2004). El itinerario geográfico como recurso didáctico para la valoración del paisaje. Universidad de Murcia.

García-González, S. M. y Gabriela, M. (2014). Categorización de preguntas formuladas antes y después de la enseñanza por indagación. Praxis \& Saber, 5(10), 75-91.

Gillies, R. M. (2016). Cooperative Learning: Review of Research and Practice. Australian Journal of Teacher Education, 41(3), 39-54.

Gómez, C. J., Ortuño, J. y Miralles, P. (2018). Enseñar Ciencias Sociales con métodos activos de aprendizaje. Reflexiones y propuestas a través de la indagación. Barcelona: Octaedro.

Hernández, R., Fernández, C. y Baptista, P. (2010). Metodología de la Investigación. México DF: McGraw Hill.

Iglesias, J., González, L. y Fernández-Río, J. (2017). Aprendizaje cooperativo. Madrid: Ediciones Pirámide.

Izquierdo, T., Asensio, E., Escarbajal, A. y Rodríguez, J. (2019). El aprendizaje cooperativo en la formación de maestros de Educación Primaria. Revista de Investigación Educativa, 37(2), 543-559. 
Johnson, D. W., Johnson, R. T. y Holubec, E. J. (1999). El aprendizaje cooperativo en el aula. Buenos Aires: Paidós.

Kocabas, A. y Gokce, D. (2017). A Scale Development for Teacher Competencies on Cooperative Learning Method. Universal Journal of Educational Research 5(3), 316-324.

Laal, M. y Laal, M. (2012). Collaborative learning: What is it? Procedia. Social and Behavioral Sciences, 31, 491-495.

Lata, S. y Castro, M. (2016). El Aprendizaje Cooperativo, un camino hacia la inclusión educativa. Revista Complutense de Educación 27(3), 1085-1101.

Liceras, A. (2018). Los itinerarios didácticos en la enseñanza de la geografía. Reflexiones y propuestas acerca de su eficacia en educación. UNES. Universidad, Escuela y Sociedad, 5, 66-81.

López, R., Miralles, P., Prats, J. y Gómez, C. (2017). Educación histórica y desarrollo de competencias. En R. López, P. Miralles, J. Prats (Dirs.) y C. Gómez (Coord.), Enseñanza de la historia y competencias educativas (pp. 7-22). Barcelona: Graó.

López de Sosoaga, A., Ugalde, A. I., Rodríguez, P. y Rico, A. (2015). La enseñanza por proyectos: una metodología necesaria para los futuros docentes. Revista Opción, 31, 395-413.

Martín, M. y Cuenca, J. M. (2011). La enseñanza y el aprendizaje del patrimonio en los museos: la perspectiva de los gestores. Revista de Psicodidáctica, 6(1), 99-122.

Molina, M. P. (2017). La aplicación de Google Earth para la Educación Patrimonial en Ciencias Sociales. Reidocrea, 18, 221-228.

(2018). La educación patrimonial en la didáctica de las ciencias sociales en primaria. Contextos Educativos. Revista de Educación, 22, 199-213.

Molina, A., Miralles, P., Deusdad, B. y Alfageme, M. B. (2017). Enseñanza de la historia, creación e identidades y prácticas docentes. Profesorado: revista de currículum y formación del profesorado, 21(2), 331-354.

Navarro, I., González, C., López, B. y Contreras, A. (2019). Aprendizaje cooperativo basado en proyectos y entornos virtuales para la formación de futuros maestros. Educar, 55(2), 519-541.

Palomares, D. y Chisvert, M. J. (2016). Cooperative learning: a methodological innovation in teacher training/El aprendizaje cooperativo: una innovación metodológica en la formación del profesorado. Cultura y Educación, 28(2), 378-395.

Payà, M., Gros, B., Piqué, B. y Rubio, L. (2018). Co-creación y validación de instrumentos para la integración del conocimiento experiencial en la formación de maestros. Profesorado. Revista de Currículum y Formación del Profesorado, 22(1), 441-460.

Perrenoud, P. (2004). Diez nuevas competencias para enseñar. Barcelona: Graó.

Prats, J. (2008). Memoria histórica versus historia enseñada. Íber. Didáctica de las Ciencias Sociales, Geografía e Historia, 55, 5-8.

Pujolàs, P. (2008). El aprendizaje cooperativo. Barcelona: Graó.

Riera, G. (2011). El aprendizaje cooperativo como metodología clave para dar respuesta a la diversidad del alumnado desde un enfoque inclusivo. Revista Latinoamericana de Educación Inclusiva, 5(2), 113-149.

Saiz, C. y Rivas, S. F. (2012). Pensamiento crítico y aprendizaje basado en problemas. Revista de Docencia Universitaria, 10(3), 325-346.

Salinas, J., de Benito, B. y Lizana, A. (2014). Competencias docentes para los nuevos escenarios de aprendizaje. Revista Interuniversitaria de Formación del Profesorado, 28(1), 145-163.

Sanchidrián, C. (2011). El uso de imágenes en la investigación histórico-educativa. Revista de Investigación Educativa, 29(2), 295-309. 
Slavin, R. (2014). Cooperative learning and academic achievement: Why does groupwork work? Anales De Psicologia, 30, 785-791.

Solaz, J., Sanjosé, V. y Gómez, A. (2011). Aprendizaje basado en problemas en la Educación Superior: una metodología necesaria en la formación del profesorado. Didáctica de las Ciencias Experimentales y Sociales, 25, 177-186.

Vicent, N. y Luna, U. (2019). Patrimonializar lo (des)conocido. Un proyecto para la formación del profesorado de Educación Primaria en torno al Patrimonio. ENSAYOS, Revista de la Facultad de Educación de Albacete, 34(1), 41-53.

Vilarrasa, A. (2003). Salir del aula. Reapropiarse del contexto. Íber: Didáctica de las ciencias sociales, geografía e historia, 36, 13-25.

Zabala, A. y Arnau, L. (2014). Métodos para la enseñanza de las competencias. Barcelona: Graó.

Zariquiey, F. (2016). Cooperar Para Aprender. Transformar el aula en una red de aprendizaje cooperativo. Madrid: Editorial SM. 